\title{
Transdisciplinarity Needs Systemism
}

\author{
Wolfgang Hofkirchner \\ Bertalanffy Center for the Study of Systems Science; Paulanergasse 13, 1040 Vienna, Austria, \\ wolfgang.hofkirchner@bcsss.org \\ Academic Editors: Gianfranco Minati, Eliano Pessa and Ignazio Licata \\ Received: 1 November 2016; Accepted: 10 February 2017; Published: 16 February 2017
}

\begin{abstract}
The main message of this paper is that systemism is best suited for transdisciplinary studies. A description of disciplinary sciences, transdisciplinary sciences and systems sciences is given, along with their different definitions of aims, scope and tools. The rationale for transdisciplinarity is global challenges, which are complex. The rationale for systemism is the concretization of understanding complexity. Drawing upon Ludwig von Bertalanffy's intention of a General System Theory, three items deserve attention-the world-view of a synergistic systems technology, the world picture of an emergentist systems theory, and the way of thinking of an integrationist systems method.
\end{abstract}

Keywords: systems thinking; systems science; systems practice; way of thinking; world picture; world-view; integrationism; emergentism; synergism

\section{Introduction}

Since we live in an age of global challenges, the responsibility of the members of the scientific community is a topical issue. The trend towards transdisciplinary studies can be seen in that context. Many researchers devoted to transdisciplinarity use systems terms but may fail in a sound usage of the terms. The further development of systems thinking, systems sciences and systems practice should address that demand. The paper at hand offers three clear-cut specifications of the systems paradigm that might support the transdisciplinary impetus.

\section{Aims, Scope, and Tools of Science}

From a philosophy of science perspective, sciences can be classified according to three dimensions of knowledge: the technological (praxiological), the theoretical (ontological), and the methodological (epistemological) dimension.

Technology is objectivated knowledge about objects for objectives. Theory is objectivated knowledge about objects. Methodology is knowledge of how to objectivate knowledge, that is, to process it in a social procedure such that it is not only subjective but might be used as an objective basis for knowledge about objects, which is required for fulfilling objectives.

Technology incorporates the aims of scientific studies; it directs theory towards practical application. Applications intervene in the real world so as to help solve problems. Problems stand at the beginning of any science because they form ends for any science. Problems are, in the last resort, social. Sciences provide the means to reach a goal, given a point of departure.

Theory embraces the scope of scientific studies; it gives deep insights in the functioning of the real world-insights that can be functionalised for the solution of problems, by informing the practice about the way to a goal from a point of departure.

Methodology provides the tools of scientific studies; it is a framework through which understanding of the functioning of the real world can be generated to serve its function during problem-solving. 


\section{Transcending the Disciplines}

Scientific disciplines are determined by specific aims, by a specific scope and by specific tools. The objective is a determinate problem solution, the object of study is a determinate piece of reality, and the objectivation is guided by a determinate mixture of methods.

However, given the rise of complex problems, monodisciplinary approaches do not fit the situation any more. Multi-, inter- and transdisciplinary approaches are needed. Transdisciplinarity has been gaining considerable attention since. It differs from monodisciplinarity in aims, scope, and tools.

As to the aims, disciplines shall be transcended by the inclusion of stakeholders through participation in the processes of research and development as well as through diffusion of innovations, which allows them to co-determine what shall be regarded as a problem and what shall be regarded as a solution. By doing so, technological knowledge shall be constructed for solving problems that are complex.

As to the scope, disciplines shall be transcended by the inclusion of interdependencies between factors across space (long-range effects), time (long-term effects), and matter (side effects) in the focus of the study. By doing so, theoretical knowledge shall be enabled to depict a bigger picture than mere isolated pieces of reality to underpin complex problem-solving.

As to the tools, disciplines shall be transcended by the inclusion of a common code that shall perform the translation of concepts of one domain to those of other domains. By doing so, methodological knowledge shall orient towards the identification of similarities across domains to gain a deeper understanding of complex problems.

\section{The Systems Paradigm}

So far, mainstream disciplines have been regarding systems practice, systems sciences, systems thinking—subsumed here under the term "systemism" (Mario Bunge)—as a determinate approach that aims at managing, intervening in, transforming, engineering or designing systems (systems technologies), which presupposes real-world systems, their dynamics and structures, or their elements as in the scope of sciences (systems theories), which, in turn, presupposes tools that frame empirical data as systems phenomena (systems methods). As such, systemism might or might not be the means of choice.

Figure 1 gives a sketch of systemism that is inspired by Robert Rosen's conception of the modelling relation. His drawing shows the natural system, that is, the object to be modelled, on the left side and the formal system, that is, the model, on the right side. From the left side to the right side, there is an encoding arrow, and from the right back to the left, a decoding arrow. After Rosen, the model is an attempt to formalise causations in the object to be modelled as inferences, whereas the encoding and decoding processes cannot be formalised themselves but make modelling "as much an art, as it is a methodology" [1] (p. 54). In Figure 1 the natural system is specified as a real-world system of any kind, and the formal system as those sciences that follow a systems approach. Rosen's encoding process is turned into a framing process and his decoding process into a designing process, both of which involve creative acts. Inside the systems, sciences layers are added to represent aims, scope and tools. The arrows between those layers do not represent entailment operations but operations that involve creative acts as well in order to accomplish the jumps between systems methods, theories and technologies.

However, systemism is not merely an option that can be taken up according to the researchers' predilection. It is far more a well-founded approach best suited for cases in which transdisciplinarity is needed. This holds not only for particular projects. It has the potential to give the whole edifice of sciences a new shape. 


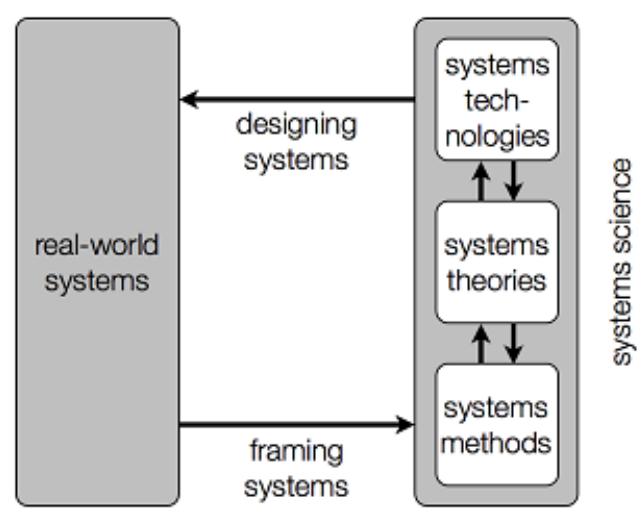

Figure 1. Modelling Systems.

The customary description of sciences involves (see Figure 2)

(a) philosophy on the uppermost general level;

(b) formal sciences, real-world sciences, and applied sciences on a next level and

(c) subgroups of the former-as logic and mathematics in the case of formal sciences, as natural sciences such as physics, etc., and social and human sciences such as sociology, etc., in the case of real-world sciences, and as engineering science (that develops technological innovations) such as computer science, etc., and management science and science of arts (that develop social innovations) in the case of applied sciences-and

(d) their sub-disciplines and sub-sub-disciplines, and so forth, on ever more specific levels.

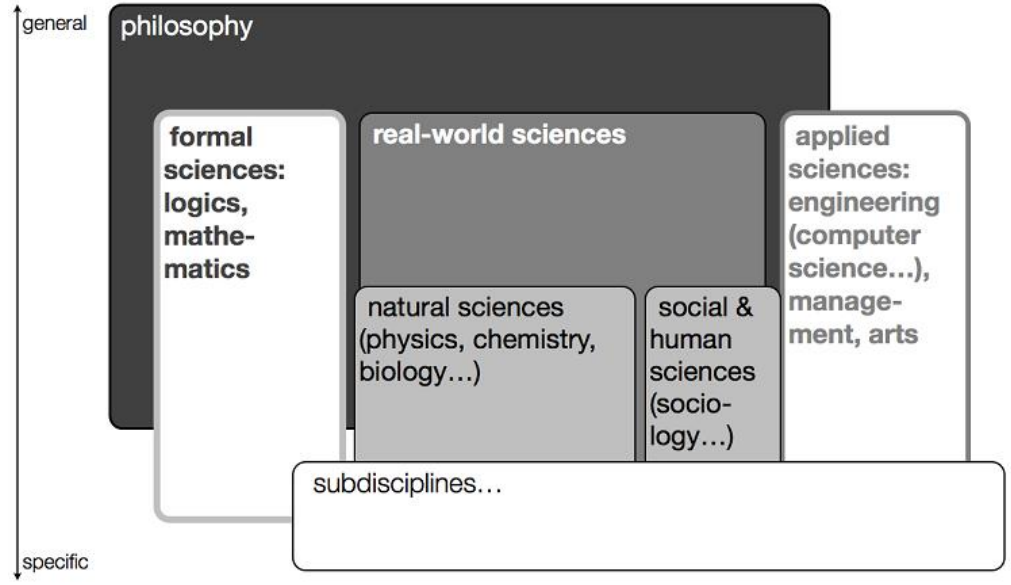

Figure 2. The Edifice of Sciences from a positivistic perspective.

Those sciences are imagined to have well-defined boundaries and to interact at best without undergoing fundamental changes themselves. Thus is the image of positivist sciences.

Systemism comes with a new understanding of sciences (see Figure 3). The systemist conception of sciences assumes semi-permeable boundaries and upward and downward interactions across the levels between all sciences. This is substantiated through defining any science in the context of discovering, describing and dealing with an overall systemic interconnectedness. Accordingly, formal sciences become part of a systems methodology that embraces formal and non-formal methods to understand systems features; real-world sciences turn into a science of different real-world systems that can be categorised as material systems, living (material) systems and social (living) systems, and 
applied sciences turn out to be a science of the artificial design of those systems. Any science is open to further specifications of subsystems, sub-subsystems and so on.

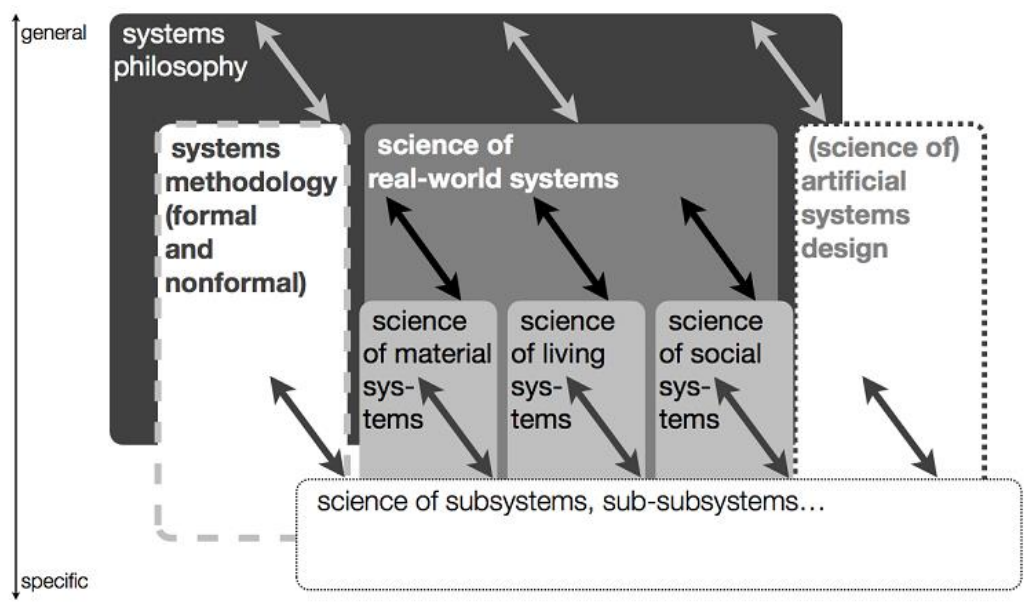

Figure 3. The Edifice of Sciences from a systems perspective.

Thus a lively body of scientific knowledge can be considered as growing and developing along the feed-forward and feedback cycles in the direction of new generalisations (upward) and new specifications (downward), while consistency is taken care of and the silos of the disciplines in the positivist perspective are broken up for the benefit of a convergent whole of science.

Systemism has been effecting a paradigm shift that can transform positivist disciplines into parts of an overarching transdisciplinary endeavour. Of course, there have been drawbacks: the resistance of the positivist science establishment has proven strong; systemism has itself branched into a plethora of different schools. Though systems terms have flooded the disciplines, the meaning of the terms is heterogeneous. So the paradigm shift is far from being completed.

The need for science and technology responses to global challenges in an intelligent way has been supporting the attraction of transdisciplinarity among the scientific community.

Drawing on Ludwig von Bertalanffy's intention to form a General System Theory [2], as well as several other compatible insights revolving around issues such as complexity, emergence, and self-organisation, can help sharpen transdisciplinary efforts. In particular, it is these features that play an innovative part in supporting the transdisciplinary agenda: systems technologies can be characterised by a new world view, systems theories by a new world picture, and systems methods by a new way of thinking.

The discussion of these issues here will follow the systems modelling processes sketched in Figure 1 and start at the bottom level with framing systems.

\subsection{A New, Systemic Way of Thinking for Transdisciplinarity: Integrationism}

Complex problems need an epistemological approach that does justice to the complexity of reality from which systems phenomena emanate. In many cases, if not in any case, an assumption has to be made about which is the interrelation of phenomena of different degrees of complexity: how does the lower-complexity phenomenon relate to the higher-complexity phenomenon (and vice versa)?

This is a question of the way of thinking. There are, in principle, three (or four) possibilities [3] (see Table 1). 
Table 1. Ways of thinking.

\begin{tabular}{|c|c|c|c|}
\hline & & Complexities & Identity and Differences \\
\hline \multirow[b]{2}{*}{ universalism } & reductionism & $\begin{array}{l}\text { levelling down } \\
\text { higher complexity }\end{array}$ & $\begin{array}{l}\text { identity at the cost of differences } \\
\text { (uniformity not diversity) }\end{array}$ \\
\hline & projectionism & levelling up lower complexity & $\begin{array}{l}\text { identity for the benefit of one difference } \\
\text { (uniformity not diversity) }\end{array}$ \\
\hline \multicolumn{2}{|c|}{ particularism: disjunctionism } & $\begin{array}{l}\text { segregating degrees } \\
\text { of complexity }\end{array}$ & $\begin{array}{l}\text { any difference at the cost of identity } \\
\text { (singularity or duality/plurality not unity) }\end{array}$ \\
\hline \multicolumn{2}{|c|}{ systemic framing: integrationism } & $\begin{array}{l}\text { conjoining complexity degrees } \\
\text { of several levels through } \\
\text { integration and differentiation }\end{array}$ & $\begin{array}{c}\text { identity and differences united } \\
\text { (dialectic of unity through diversity, } \\
\text { including as less unity as necessary and as } \\
\text { much diversity as possible) }\end{array}$ \\
\hline
\end{tabular}

First, there is a universalist way of thinking that gives priority to uniformity over diversity. It comes in two varieties:

(a) the levelling down of phenomena of higher complexity to phenomena of lower complexity; identity of the phenomena is established at the cost of differences; this is known as reductionism;

(b) the levelling up of phenomena of lower complexity to phenomena of higher complexity; identity of the phenomena is established for the benefit of one difference; this is called "projectionism"; higher complexity is erroneously conceptualised at a level where it does not exist.

The second way of thinking is a particularist one. Priority is given to the singularity of a difference or the plurality of all differences over unity. The disjoining of phenomena of different degrees of complexity establishes the identity of a particular difference or identities of any difference, thus an equivalence of differences-indifference-at the cost of an identity common to the phenomena. That is called "disjunctionism".

A third option is that way of thinking that inheres in systemism. It negates universalism and particularism as well and interrelates phenomena to each other through integration and differentiation of their complexity degrees. The union of identity and differences yields unity through diversity. That is the meaning of integrationism here. That is, the phenomenon with a lower degree of complexity shares with the phenomenon with a higher degree of complexity at least one property, which makes both of them, to a certain extent, identical, but the latter phenomenon is in the exclusive possession of at least another property, which makes it, to a certain extent, distinct from the former. So both phenomena are identical and different at the same time.

The method of transdisciplinarity can take advantage of bringing this new systems method to bear: framing the phenomena through the equilibration of integration and differentiation during the processes of conceptualisation in order to rule out reductionist, projectionist, and disjunctionist ways of thinking.

Example. Let us take the relationship of social science and engineering science as an example for how to transcend the borders of both disciplines by making use of a systemic framing and transform their relationship into a true transdisciplinary one (see Table 2). 
Table 2. Ways of thinking in society and technology.

\begin{tabular}{|c|c|c|c|}
\hline & & Complexities & Identity and Differences \\
\hline \multicolumn{2}{|c|}{ technomorphism } & man is deemed a machine & $\begin{array}{l}\text { the conception of the artificial is sufficient } \\
\text { for comprehending social forms }\end{array}$ \\
\hline \multicolumn{2}{|c|}{ anthropo-socio-morphism } & $\begin{array}{l}\text { the machine is } \\
\text { deemed man-like }\end{array}$ & $\begin{array}{l}\text { the conception of social forms is necessary } \\
\text { for comprehending the artificial }\end{array}$ \\
\hline \multirow{3}{*}{ hetero-morphism } & socio-centrism & man is deemed exclusive & $\begin{array}{l}\text { social forms are construed as distinctive } \\
\text { over and against the artificial }\end{array}$ \\
\hline & techno-centrism & $\begin{array}{l}\text { the machine is } \\
\text { deemed exclusive }\end{array}$ & $\begin{array}{l}\text { the artificial is construed as distinctive over } \\
\text { and against social forms }\end{array}$ \\
\hline & relativism & $\begin{array}{l}\text { man and machine are } \\
\text { deemed apart }\end{array}$ & $\begin{array}{l}\text { both social and artificial forms are } \\
\text { construed side by side }\end{array}$ \\
\hline \multicolumn{2}{|c|}{$\begin{array}{l}\text { systemic framing: } \\
\text { techno-social systems }\end{array}$} & $\begin{array}{l}\text { man and machine are } \\
\text { deemed nested }\end{array}$ & $\begin{array}{l}\text { the understandings of social and artificial } \\
\text { forms join into the understanding of } \\
\text { techno-social systems }\end{array}$ \\
\hline
\end{tabular}

In order to combine social science with engineering science, representatives of the latter might be inclined to reduce that which is human to that which is engineerable: man is deemed a machine. Operation Research, Cybernetics, Robotics, Mechatronics, the fields of Artificial Intelligence and so-called Autonomous Systems, among others, are liable to cut the understanding of man who is a social being free from the understanding of social relations; the conception of the human body free from the conception of individual actors; and conceiving of mechanics free from conceiving of the organism. Mechanical architectures and functioning that are constituents among others of human life structures and processes are analysed and hypostatised as sufficient for the comprehension of man ("technomorphism").

Or representatives of social sciences-not unlike those of other disciplines-might share a predilection to understand the whole world, including artifactual mechanics, by projecting characteristics of the social world onto the former: the machine is deemed man-like. Actor-Network Theory, Deep Ecology, New Materialism, Info-Computationalism and others are prone to blur the boundaries between the social sciences and the sciences of living things, between the latter and the sciences of physical things and, eventually, between the sciences of physical things and engineering sciences by attributing human features to any of those non-social disciplines. The conception of social forms is thought necessary for the comprehension of everything. That is blunt anthroposociomorphism.

Segregation might be made for the sake of either the identity of social science or that of engineering science: anthropocentric or, better, sociocentric positions traditionally distinguish the investigation of man as exclusive and belittle engineering undertakings, whereas trans- and post-humanistic positions argue for an imminent advent of a technological singularity that will make machines outperform man and thus the human race obsolescent. However, segregation might also promote the juxtaposition of social science and engineering science: they co-exist, any of them is worth as much as the other, and there are no grounds for giving supremacy to what counts as social forms or what counts as technological forms (relativism). Both are conceived as reciprocally exclusive ("heteromorphism").

No one of these options does establish true transdisciplinarity. In the case of technomorphism, social science gives up any autonomy and is invaded by engineering science. In the case of anthroposociomorphism, any autonomy of engineering science is forfeited, as social science projects its autonomy onto engineering science. In the case of heteromorphism, each discipline claims full autonomy over its own, self-contained area. A way out can be seen through an approach that assumes an interrelation of both disciplines in a systemic framework that grants (relative) autonomy to each of them according to their place in the overall framework. Both disciplines complement each other for the sake of a greater whole. That greater whole is achieved by framing both disciplines in a systems perspective, that is, by framing them as part of systems science. As such, social and 
engineering sciences combine for a common understanding of the systemic relationship of society and technology - of emerging techno-social systems. They make use of systems methodologies for empirically studying social systems and the artifactual in the context of technological applications implemented by social systems design. By doing so, they can form a never-ending cycle in which each of them has a determinate place: social systems science can inform engineering systems science by providing facts about social functions in the social system that might be supported with technological means; engineering systems science can provide technological options that fit the social functions in the envisaged techno-social system; social systems science can, in turn, investigate the social impact of the applied technological option in the techno-social system and provide facts about the working of technology. The social and the engineering parts of techno-social systems science are coupled so as to promote an integrated technology assessment and technology design cycle in a transdisciplinary sense.

\subsection{A New, Systemic Picture of the World for Transdisciplinarity: Emergentism}

Systems theories provide an ontology in which complex problems are pictured as complex because they take part in an overall interconnectedness of processes and structures that are constituted by self-organising real-world systems. Those systems bring about evolution and nestedness as emergent features of reality.

The world is pictured according to a multi-stage model of evolutionary systems [4] (see Figure 4).

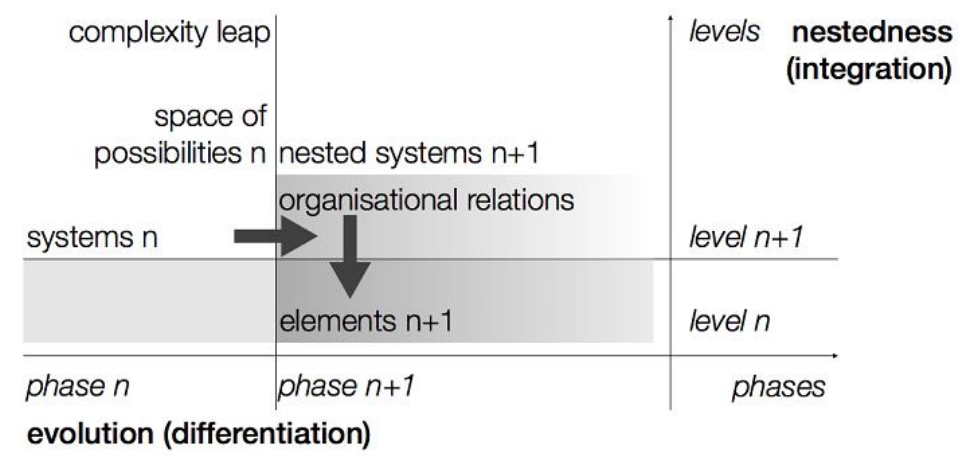

Figure 4. The multi-stage model of evolutionary systems.

Systems evolve during a phase $n$. Spontaneously, at a certain point in time, a leap in complexity emerges and one possibility out of the space of possibilities that are rooted in the reality of the systems during the phase $n$ (which form the necessary condition for the transition to phase $n+1$ ) is realised such that new organisational relations emerge. Those organisational relations realise a higher order in that they nest the old systems $n$ as elements $n+1$ of the new systems $n+1$ during the phase $n+1$. Thus they form another level $n+1$ above the level $n$ that is being reontologised, reworked, reshaped. Emergence of new systems in the course of evolution (differentiation) entails dominance of higher levels through the reconfiguration of what is taken over from the old (integration).

Emergentism (another term coined by Bunge) is an important ingredient of systems theories. It helps understand events and entities that function as less than strict deterministically. Transdisciplinarity must take into consideration less-than-strict determinism, which means that the mechanisms of the real world are not machine-like.

Emergentism provides an ontological superstructure for epistemological integrationism. Integrationism can integrate because evolution lets new features emerge.

Example. Let us consider the issue of anthroposociogenesis [5] as an example for the transcendence of natural and cultural science towards a true transdiscipline of anthropology by a systemic image of the evolutionary formation of society and humans.

In naturalistic accounts, the advent of human culture is owed to the development of nature. The former is the necessary result of the latter. Anatomic features such as cerebral growth, the descent 
of the larynx, bipedalism and the opposable thumb and the fingers, among others, are named as biological traits that seem to have caused psychic functions such as thought, the ability to speak and the ability to devise and manufacture artefacts, in particular tools.

One variety of culturalism would conflate the distinction between nature and culture as well, postulating that cultural features occur in pre-human life and overemphasising the continuity from natural to cultural evolution.

Another variety of culturalism would postulate a discontinuity between nature and culture and deny any comparability. Both spheres are considered to be ontologically independent of each other, and therefore, on that basis they might interact.

In contradistinction, emergentism sees nature as the necessary condition upon which culture as an evolutionary contingency could emerge. In turn, culture, as a supra-system, began to dominate via the higher-order structure from which it had taken its departure (see Figure 5). The key idea is that it is possibilities of co-operation that, when realised, made the difference in evolution. Evolutionary pressure unfolded a ratchet effect that yielded ever higher complex cooperation. Michael Tomasello characterises the life of our great ape ancestors as "mostly individualistic and competitive" [6] (p. 4). The ancestors were "individually intentional and instrumentally rational" [6] (p. 30) as great apes are still today. It was not before taking advantage of going beyond "individual intentionality" and adopting "more complex forms of cooperative sociality" [6] (p. 31) that early humans began to speciate. This brought about "shared intentionality" and enabled them to achieve shared goals. In particular, Tomasello hypothesises two steps towards a differentiated shared intentionality. A first step occurred "in the context of collaborative foraging" [6] (p. 33) around two million years ago with a culmination 400,000 years ago. This was a step from individual intentionality to joint intentionality, from the competitive sociality of great ape ancestors to ad hoc dyadic relationships with a "significant other" (G. H. Mead). Multiple and vanishing dyadic relationships formed in which early humans shared a joint goal. A second step led from early to modern humans some 200,000 years ago. They became "thoroughly group-minded individuals" [6] (p. 80) in larger groups, in which they "had to be prepared to coordinate with anyone from the group, with some kind of generic other" [6] (p. 81). This was a step from joint intentionality to collective intentionality, a step to the co-operation within a larger group organisation (culture) as the "generalised other" (Mead). Every step was a step of emergence of social systems in a new quality owed to the contingent realisation of possible social relations that represent a higher order of cooperation and reconfigure the old system. By accepting the interplay of emergence and dominance, the objects of natural science and cultural science reveal their systemic interrelationship and give way to a true transdisciplinary theory of the origins (and evolution) of human, social systems.

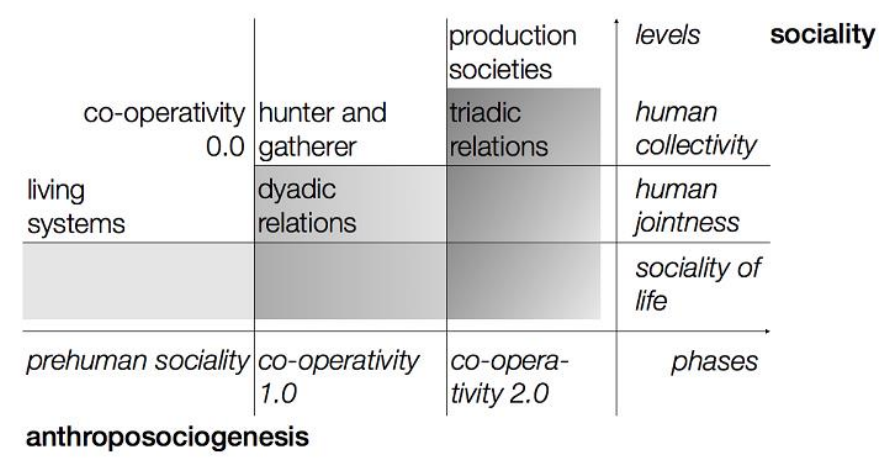

Figure 5. The emergence of cultural systems from natural systems through relations of joint cooperation and collective cooperation. 


\subsection{A New, Systemic World View for Transdisciplinarity: Synergism}

Acting in the face of complex problems is based on praxiological assumptions about the interference with self-organising systems. Known mechanisms can be furthered or dampened according to what the goal shall be.

In the course of evolution, systems move on trajectories on which bifurcations occur. Bifurcations come up with a variety of possible future trajectories. Systems might not be in the position to avert devolution (a path that leads to the breakdown of the system) or they might be able to achieve a leap from the previous level of evolution on which they could enjoy a steady state onto a higher level which forms part of a successful mega-evolution (a breakthrough to a path that transforms the system) [7] (p. 314) [8] (pp. 103-104). Amplified fluctuations of parameters indicate possible and necessary punctuations (see Figure 6).

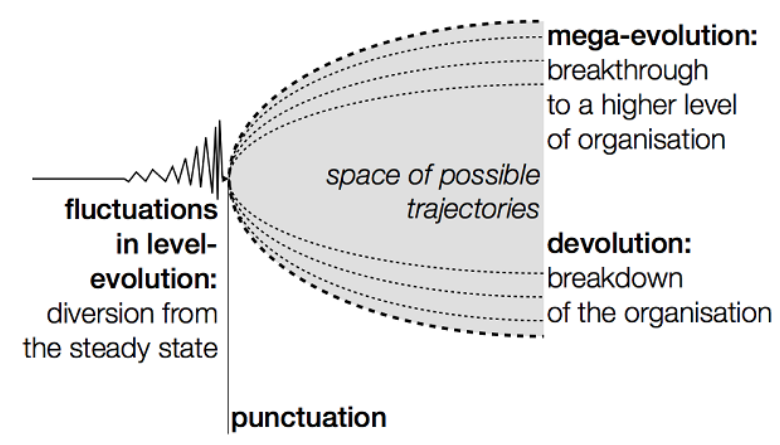

Figure 6. Bifurcations in systems evolution.

Self-organising systems have as raison d'etre the provision and production of synergetic effects [9]. If the organisational relations are not able any more to provide and help the elements produce synergy, the system will break down. Hindrances of letting synergy emerge are called frictions. Any social system is a social system by virtue of organisational relations of production and provision of the common good, that is, the commons are the social manifestation of synergy [10]. Hindrances of the commons supply are frictions that are systemic dysfunctions due to the suboptimal organisation of the synergetic effects. Any meaningful technology is oriented towards the alleviation of frictions and the advancement of synergy.

Thus systems technologies can help orient transdisciplinarity. Meaningful technology is technology endowed with meaning by

(1) the participation of those affected in an integrated technology assessment and design process (that is, design builds upon assessment);

(2) for the reflection of the expected and actual usage of technology: the assessment and design criterion is social usefulness, that is, the reflection of both;

(a) the adequacy to the purpose (utility; operational knowledge: know-how) and

(b) the purpose itself (the function technology serves; orientational knowledge: know why and what for).

The purpose is advancing the commons.

Synergism, the orientation towards synergy for every real-world system and towards the human value of the commons in the case of social systems-which is a world-view (weltanschauung) because it is value-laden-is the praxiological superstructure for emergentism. Synergy emerges, emergence brings about synergy.

Example. Let us turn to the crises that manifest themselves in the tension between social systems on the level of today's nation states as well as within every such social system regarding their 
subsystems such as, in particular, the cultural, the political, the economic, the ecological (eco-social) and the technological (techno-social) subsystems, which means that several branches of social sciences are challenged. The critical situation might be complicated or complex to analyse. Sometimes it is said that complicatedness - the presence of many factors that influence each other - is not an obstacle that we would not be able to overcome. However, the opposite might be true. The huge amount of options of interventions-proposed by different disciplines-might actually pose a practical difficulty. However, on the other hand, the situation we face nowadays is rather complex. In addition, if complexity enters the stage, problem-solving is also possible despite opposite views-in a true transdisciplinary effort.

Today, enclosures of the commons have been aggravated to such a degree that all of them morphed into global challenges. Global challenges drive an accumulation of crises that mark a decisive bifurcation. A Great Bifurcation lies ahead of humanity (see Figure 7) [11].

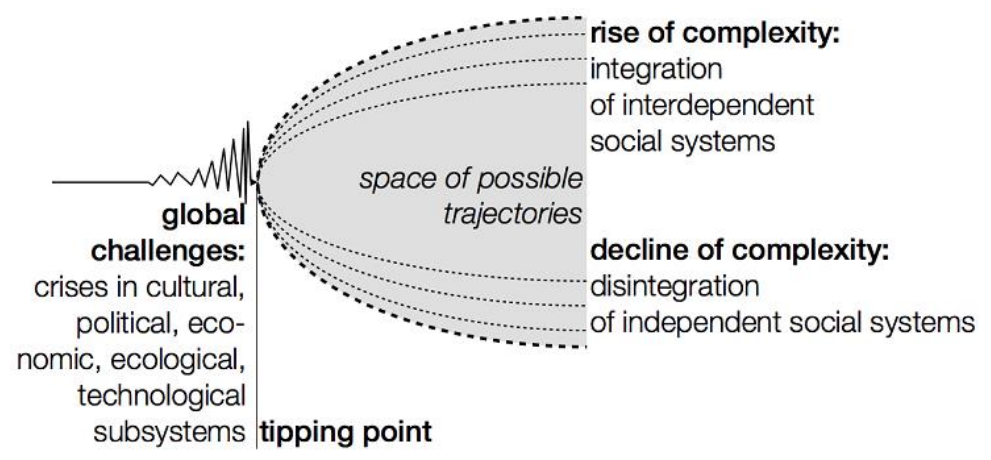

Figure 7. The Great Bifurcation in social systems evolution.

On the one hand, a possible change never seen before might be imminent-a transformation of the current state of civilisation into a new state that brings about a peaceful, environmentally sound and socially and economically just and inclusive world society by the integration of differentiated interdependent social systems. That rise in complexity is possible. If there is a mismatch between the complexity of a system and the complexity of the problems faced by the system, that system can catch up. It can solve the problem by activating the collective intelligence of the co-systems it is made up of and raise the complexity of its organisational relations or by activating the collective intelligence of co-systems of its own and raise the complexity of that supra-system in which they are nested in order to match or surpass the complexity faced. Intelligence is the capability of self-organising systems to generate that information which contributes in the best way to solving problems. The better their collective intelligence, that is, the better their problem-solving capacity and the better their capability to generate information, the better their handling of the crisis and the order they can reach. Higher complexity not only signifies a higher degree of differentiation. At least as importantly, it signifies a new quality of integration. Only a new level of integration can deal with an intensification of differentiation. The new system would disclose the commons. "It would be a Global Sustainable Information Society (GSIS).

GSIS requires,

(1) for the first time in the history of our planet, on a higher social level — that is, globally-

(2) a reorganisation of the social relations within and in between the interdependent social systems such that sociogenic dysfunctions with respect to the social, the social-ecological and the socio-technological realms can be contained-that is, a transformation into sustainable development -

(3) through conscious and conscientious actors that are not only self- but also community-concerned — that is, under well-determined informational conditions." [11] (15) 
On the other hand, a decline in complexity might be imminent, eventually leading to disintegration of allegedly independent social systems. Civilisation would fall apart.

Systemism is key to tackling complex problems such as the reciprocal influences of varying kinds of factors dealt with on the basis of varying disciplinary backgrounds within the social sciences in a transdisciplinary way. It is thus key to tackling global challenges and guiding the transformation of our social systems onto the next level of cooperation through a social systems design based upon social systems technologies.

\section{Conclusions}

In summary, systemic transdisciplinarity

(1) aims - by a systems world-view—at providing scientific knowledge for solving problems of frictions in the functioning of real-world systems, in particular in processes of the provision and production of the commons in social systems through meaningful systems technologies that support the re-organisation of social systems in order to safeguard sustainable development and rule out self-inflicted breakdowns;

(2) has as its scope the functioning of emergent real-world systems in the interconnectedness of their evolution and their nestedness, the scientific knowledge of which is a theoretical systems world picture needed for alleviating frictions;

(3) uses tools that generate scientific knowledge through a systems way of thinking by the method of equilibrating integration and differentiation for a proper understanding of how complexity grows.

Systemism has the power to transform the disciplines.

Conflicts of Interest: The author declares no conflict of interest.

\section{References}

1. Rosen, R. Life Itself; Columbia University Press: New York, NY, USA, 1991.

2. Bertalanffy, L.V. General System Theory; With a Foreword by Wolfgang Hofkirchner \& David Rousseau; George Braziller: New York, NY, USA, 2015.

3. Hofkirchner, W. Four ways of thinking in information. Triple-C: J. Glob. Sustain. Infor. Soc. 2011, 9, 322-331.

4. Hofkirchner, W. Emergent Information, a Unified Theory of Information Framework; World Scientific: Singapore, 2013.

5. Hofkirchner, W. Ethics from Systems: Origin, Development and Current State of Normativity. In Morphogenesis and the Crisis of Normativity; Archer, M.S., Ed.; Springer: Dordrecht, The Netherlands, 2016; pp. 279-295.

6. Tomasello, M. A Natural History of Human Thinking; Harvard University Press: Cambridge, MA, USA, 2014.

7. Haefner, K. Information Processing at the Sociotechnical Level. In Evolution of Information Processing Systems; Haefner, K., Ed.; Springer: Berlin, Germany, 1992; pp. 307-319.

8. Oeser, E. Mega-Evolution of Information Processing Systems. In Evolution of Information Processing Systems; Haefner, K., Ed.; Springer: Berlin, Germany, 1992; pp. 103-111.

9. Corning, P. The Synergism Hypothesis; McGraw-Hill: New York, NY, USA, 1983.

10. Hofkirchner, W. Creating Common Good. The Global Sustainable Information Society as the Good Society. In Morphogenesis and Human Flourishing; Archer, M.S., Ed.; Springer: Dordrecht, The Netherlands, 2017; in print.

11. Hofkirchner, W. Information for a Global Sustainable Information Society. In The Future Information Society, Social and Technological Problems; Hofkirchner, W., Burgin, M., Eds.; Wolrd Scientific: Singapore, 2017; pp. 11-33. 